\title{
Symmetrical Graph Neural Network for Quantum Chemistry with Dual Real and Momenta Space
}

\author{
Shuqian Ye, Jiechun Liang, Rulin Liu, Xi Zhu* \\ Shenzhen Institute of Artificial Intelligence and Robotics for Society (AIRS) \\ The Chinese University of Hong Kong, Shenzhen \\ 14-15F, Tower G2, Xinghe World, Rd Yabao, Longgang District, \\ Shenzhen, Guangdong, China, 518172 \\ Email: zhuxi@cuhk.edu.cn
}

\section{Supplementary Materials}

\section{Section S1. The flow of the SY-GNN model}

The SY-GNN model uses atomic numbers $Z$ and atomic coordinates $r$ as inputs. The main algorithm can be summarized as follows:

1) Use group theory to find out the symmetric units and equivalent atoms of the symmetrical molecule.

2) Encode each atom in the molecule into the feature space.

3) Based on equivalent atoms information, the interaction module updates the feature of atoms in the symmetric unit.

4) Concatenate the input and output of the interaction module and share the feature among equivalent atoms.

5) Repeat steps 3 and 4 according to the number of interactive modules.

6) Use self-attentive readout to aggregate the features of all atoms in the molecules.

7) Use different output layer to output the predicted result.

8) Calculate the loss between the predicted value and ground truth. (Only for training phase) 
9) Use the multi-tasks optimizer to calculate the gradient from the loss. (Only for training phase)

\section{Section S2. Graph Theory Layer}

In our work, the rotation symmetry is considered. There are three primary symmetries in the QM-sym database, including $\mathrm{C}_{2}, \mathrm{C}_{3}$, and $\mathrm{C}_{4}$. The graph theory layer is a non-parametric algorithm which can determine the symmetry of the input molecule. Since the molecule is accurate in the dataset, our group theory based algorithm can determine the correct symmetry surely.

As for a space-related property, when the algorithm wants to find out the symmetry of a molecule, the first thing is to check whether this molecule has a symmetric unit. This job can be determined by counting the number of each kind of atoms. If the numbers are multiples of 2,3 , or 4 , it is possible to be a symmetrical molecule. The next step is to discover the rotation plane, and the final step is to determine the rotation axis. Around the axis, we can check that for a specific atom, whether the same element exists on the target positions if the molecule has $\mathrm{C}_{2}, \mathrm{C}_{3}$, and $\mathrm{C}_{4}$ symmetry. For example, if the molecule has $\mathrm{C}_{3}$ symmetry, all atoms that do not exist on the rotation axis will overlap the same element when the molecule rotates 120, 240, and 360 degrees. In this way, we can extend our method easily and accurately determine the symmetry of a molecule besides $\mathrm{C}_{2}, \mathrm{C}_{3}, \mathrm{C}_{4}$.

\section{Section S3. Details for model training}

We use a single TITAN XP graphics card for training. We develop our code for SY-GNN based on SchNetPack ${ }^{1}$ using PyTorch deep learning framework ${ }^{2}$.

All models are trained with $80 \%$ of all molecules in the QM-sym database, and then $10 \%$ of the molecules are used to validate the trained model, and the best-performing model in the validation set is selected. The remaining $10 \%$ of molecules are used to demonstrate the result in the below section. All models are trained with stochastic gradient descent using Adam ${ }^{3}$ optimizer. At the beginning of each epoch, we shuffle the molecules in the training set, to ensure the model will not memorize the order of input molecules. 
For SchNet (STL), we use 6 interaction modules, set the size of atomic representation to 64, and Gaussian interatomic distance expansion dimension to 25, each interaction module output 128 dimension features for each atom. We use an initial learning rate of $10^{-4}$ and an exponential learning rate decay with a rate of 0.96 per 5,000 back-propagation steps, and train for 300 epochs. We use 96 molecules in each mini-batch for STL task.

For FMAPP training, we follow the hyperparameters used in the official open-source code of FMAPP. The dimension of vector to represent atoms is 25 , the number of the hidden layer is 6 , the batch size is 64 , the learning rate is $10^{-3}$ with decay rate 0.95 per 10 epochs, the weight decay parameter for optimizer is $10^{-6}$ and optimize the model with 300 epochs.

For SchNet (MTL) and SY-GNN, all the configuration is the same as SchNet (STL) without declaration below. Since we need to extract features for both K and R space, while for STL task only features for single $\mathrm{K}$ or $\mathrm{R}$ space are needed, we should use a higher dimensional vector to represent the features of atoms, hence the output dimension of each interaction module is expended to 192. Limited by the graphic memory, the batch size is reduced to 64 . The hyper-parameter is obtained through a grid search method, and the hyper-parameters to obtain the lowest error are represented.

For the sum-up loss function for SchNet (MTL), the weights for different loss is variant, to balance the loss. The weight for loss on heat capacity, isotropic polarizability, electronic spatial extent, and dipole moments are $10^{-3}, 10^{-4}, 10^{-6}, 10^{3}$ individually, others are 1 .

For the multitask optimizer for SY-GNN, considering the difficulty of different tasks. For isotropic polarizability, zero point vibration energy, and electronic spatial extent, we will give extra 1, 2, and 3 iterationss in each round of optimization. Other properties will only have 1 iteration to be optimized in each round.

When previous methods trained on QM9 and other datasets, they usually used atom reference, which means the model only needs to estimate the difference between sum-up of all atoms' properties and the ground truth molecular properties for some extensive properties. But QM-sym dataset does not provide such an atom reference. We calculate the atom reference for $G, H, U, U_{0}$ using linear regression, and applied the calculated atom reference into the model on SY-GNN. For SchNet, we follow the official implementation (https://github.com/atomistic-machine-learning/schnetpack) in 
SchNetPack ${ }^{1}$ to add atom reference. For FMAPP, we also follow the official implementation (https://github.com/masashitsubaki/molecularGNN 3Dstructure) that do not use atom reference.

In Table 1, we class 12 properties into $\mathrm{K}$ space and $\mathrm{R}$ space. The classification guideline is that if the property is mainly determined by the real-space coordinates, we consider it as a $\mathrm{R}$ space property; if the property is mainly determined by electrons movement and band structure, we will put it in the K space prediction region.

\section{Section S4. Calculation of K/R space error}

The molecular symmetry in R and K (orbital symmetry) spaces are equally essential to determine the molecular properties, especially for the selection rules in the excited states. The $\mathrm{R}$ space can be considered as the position in the three-dimensional coordinate, which means the $\mathrm{x}, \mathrm{y}, \mathrm{z}$ magnitude of atoms. In this way, the properties that correlated to the atoms and phonons are considered as $\mathrm{R}$ space properties. For example, the heat capacity is mainly determined by the vibration of phonons, which will also result in the vibration of atoms, so it is an $\mathrm{R}$ space property. As for the $\mathrm{K}$ space, which is the energy space, denotes the electron orbitals of the atom. Some properties like the HOMO energy, LUMO energy, and orbital symmetry are K space properties.

Since all models except our SY-GNN are designed for datasets with small size and asymmetric molecules database like QM9, we adopt the result obtain from the QM9 dataset to calculate K/R space error for a fair comparison. The error obtains from QM9 is lower than QM-sym, since the molecules on QM-sym has more types of atoms and larger molecular size, i.e., the difficulty of prediction is much higher. The data used to calculate relative K/R space error for all models except SY-GNN and FMAPP are from Lu et al.'s paper ${ }^{4}$. The data of FMAPP is from Tsubaki et al.'s paper5.

The prediction errors of $\mathrm{U}, \mathrm{G}, \mathrm{H}$ for models $\mathrm{RF}+\mathrm{BAML}, \mathrm{KRR}+\mathrm{BOB}, \mathrm{KRR}+\mathrm{HDAD}$, and GG vibrationrelated lack, based on our experience, the prediction errors of $\mathrm{U}, \mathrm{G}, \mathrm{H}$ are very closed to the prediction error of $U_{0}$. So, for these four models, we assume the prediction error of $U, G, H$ are the same as the prediction error of $\mathrm{U}_{0}$.

There are eight properties belongs to R space, $\alpha, \mathrm{G}, \mathrm{H}, \mathrm{U}, \mathrm{U}_{0}, \mathrm{R}^{2}, \mathrm{C}_{\mathrm{V}}$, and $\mu$. All 4 properties, $\epsilon_{\mathrm{HOMO} O}$, $\epsilon_{\mathrm{LUMO}}$, gap, and ZPVE are used to calculate the K space error. 
We use $\bar{N}_{\text {avg }}$ to represent the average size of molecules in the database. For QM9, it should be about 17.98, and about 49.76 for QM-sym. We can calculate K/R space error by following formulas:

$$
\begin{gathered}
E_{R}=\frac{G+H+U+U_{0}+R^{2}+\alpha+\mathrm{Cv}+\mu}{8 \bar{N}_{\text {avg }}^{2}} \#(13) \\
E_{K}=\frac{\epsilon_{\mathrm{HOMO}}+\epsilon_{\mathrm{LUMO}}+\text { gap }+\mathrm{ZPVE}}{4} \#(14)
\end{gathered}
$$

Almost all properties in R space are extensive, so we add a term $\bar{N}_{\text {avg }}^{2}$ in the denominator to eliminate the effect of the size of molecules. We choose the power of 2 by considering the dimension of molecules in QM-Sym. Almost all properties of the K space quantities are intensive, so we do not all this term. The units for the properties follows the units shown in Table 1.

\section{Section S5. Shapes of the molecular orbitals}

The location and the shape of the $\pi$ orbitals could be determined given the irreducible representation of the corresponding orbitals. In the database utilized, a molecule whose symmetry group is $\mathrm{C}_{4 \mathrm{~h}}$ was picked as an example to show the procedure.

The example is called 1,2,3,4-tetrapropylidene-cyclobutane, as shown in Fig. 5B. The atoms on the central cyclobutene were numbered 1, 2, 3, 4, and the atoms linked to them via the double bonds were numbered $5,6,7,8$, respectively. In such a molecule, since the double bonds occur only on the atoms numbered $1,2,3,4,5,7,8$, in determining the $\pi$ orbitals, only these 8 atoms require attention. The character table and the representation of the $\pi$ bonds are as in Table S1.

Thus, according to the decomposition formula $a_{i}=\Sigma_{R} \chi_{r}(R) \chi_{i}(R) / h$, the result could be written in the form:

$$
\Gamma_{\pi}=2 A_{u}+2 B_{u}+4 E_{g} \#(15)
$$

indicating that there are two $A_{u}$ orbitals, two $B_{u}$ orbitals, and four $E_{g}$ orbitals. Based on the transformation properties and the character table of the given molecule, the orbitals could be determined as: 


$$
\begin{gathered}
A_{u}:\left\{\begin{array}{l}
\psi_{1}+\psi_{2}+\psi_{3}+\psi_{4} \\
\psi_{5}+\psi_{6}+\psi_{7}+\psi_{8}
\end{array}\right. \\
B_{u}:\left\{\begin{array}{l}
\psi_{1}-\psi_{2}+\psi_{3}-\psi_{4} \\
\psi_{5}-\psi_{6}+\psi_{7}-\psi_{8}
\end{array}\right. \\
E_{g}:\left\{\begin{array}{l}
\psi_{1}-\psi_{3} \\
\psi_{2}-\psi_{4} \\
\psi_{5}-\psi_{7} \\
\psi_{6}-\psi_{8}
\end{array}\right.
\end{gathered}
$$

Beware that the above wave equations require normalization. By putting orbitals with the same symmetry in one secular equation to solve for the eigenvalues and plotting them for the energy, with the assumption that the on-site energy $\alpha=0$, and the hopping parameter $\beta<0$, the energy level diagram could be drawn, as in Fig. 5B. In constructing the secular equations, the entries are calculated with the Hückel approximation:

$$
\psi_{i}|H| \psi_{j}=\left\{\begin{array}{lc}
\alpha, & i=j \\
\beta, & i \text { is adjacent to } j \#(17) \\
0, & \text { otherwise }
\end{array}\right.
$$

Based on the energy of the energy level, i.e., the eigenvalue of the corresponding secular equation, one could solve for the linear combination of the wave equations involved and thus determine the shape of the molecular orbitals based on the absolute value and the sign of the coefficients. Take $B_{u}$ as an example, the secular equation from the wave equations of $B_{u}$ is

$$
\begin{gathered}
{\left[\begin{array}{cc}
\frac{1}{4}(4 \alpha-8 \beta) & \frac{1}{4}(4 \beta) \\
\frac{1}{4}(4 \beta) & \frac{1}{4}(4 \alpha)
\end{array}\right]\left[\begin{array}{l}
c_{1} \\
c_{2}
\end{array}\right]\left[\psi_{B_{u}}^{1}, \psi_{B_{u}}^{2}\right]=-(1-\sqrt{2}) \beta\left[\begin{array}{l}
c_{1} \\
c_{2}
\end{array}\right]\left[\psi_{B_{u}}^{1}, \psi_{B_{u}}^{2}\right]} \\
\end{gathered}
$$




$$
\text { i.e. } c_{2}>c_{1}>0 \#(18)
$$

indicating that the wave equations of $B_{u}$ are along the same direction. Thus, as can be seen in the figure, for $B_{u}$ orbital, the atomic orbitals $\psi_{1}, \psi_{3}, \psi_{5}, \psi_{7}$ are along the same direction, while the others are opposite.

\section{Section S6. Spectral transition probability}

The transition probability of electrons between the molecular orbitals via light could be determined by the tensor product of the relative irreducible representations of the initial and final energy levels.

By picking the irreducible representations of the involved orbitals as $\psi_{i}, \psi_{j}$, and defining a transition moment operator $\mu$ for the incident light, the intensity of the transition is given by the Equation (12).

The characters of the representation of the tensor product are equal to the products of the characters for the same symmetry operation of the representations involved. Only when the symmetric operation is present in the result of $\psi_{i} \otimes \mu \otimes \psi_{j}$, will this integral be nonzero, i.e., the transition of electrons from $\psi_{i}$ orbital to $\psi_{j}$ orbital via operator $\mu$ is possible. In the case of the spectral transition of electrons, the transition moment operator is the irreducible representation with the same linear symmetry as the incident light. For example, if the molecule is of $\mathrm{C}_{4 \mathrm{~h}}$ symmetry as described by the character table above, the incident light along the $z$-direction is described by $\mu=A_{u}$. As an example, the transition property of the molecule above, from HOMO to LUMO, can be calculated as in Table S2.

As shown in the decomposition, since $\mathrm{A}_{\mathrm{g}} \mathrm{A}_{\mathrm{g}}$ occurs in only two of them decompositions, so only when

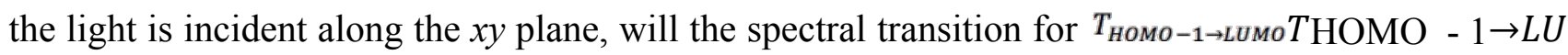
MOand $T_{\text {нимо } \rightarrow \text { LUмо }+1} T \mathrm{HOMO} \rightarrow L U \mathrm{MO}+1$ be possible. The others will not be able to transit via light along $x, y, z$ directions, but might be able to be triggered by other mechanisms. 
Figures and Tables for Supplementary Materials

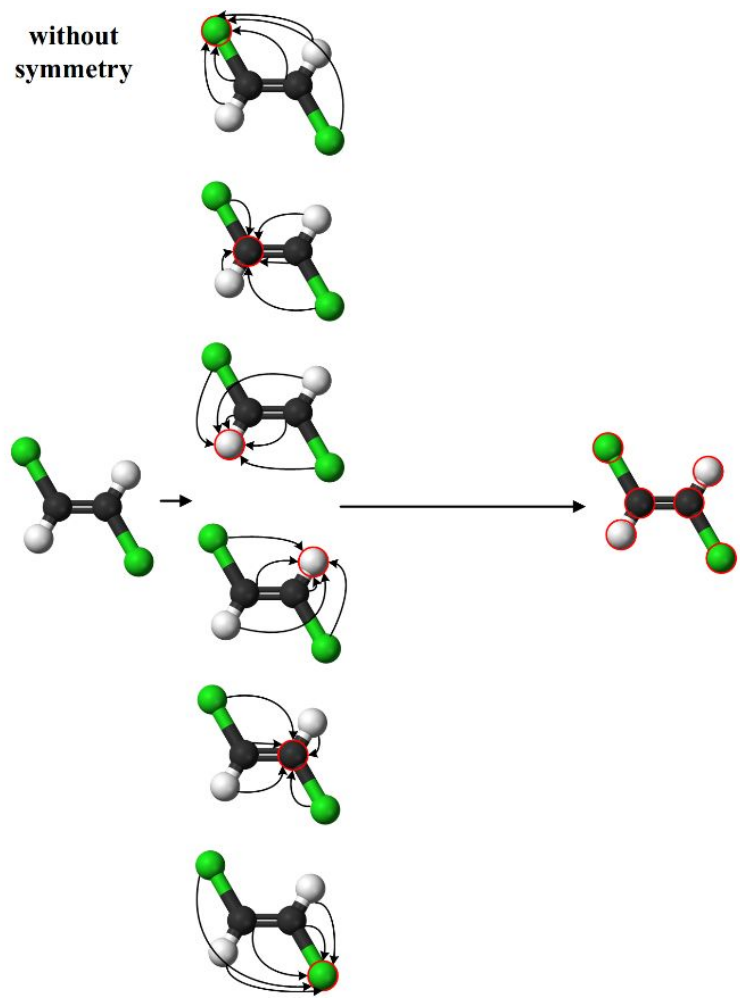

$$
\begin{aligned}
& \text { with } \\
& \text { symmetry }
\end{aligned}
$$

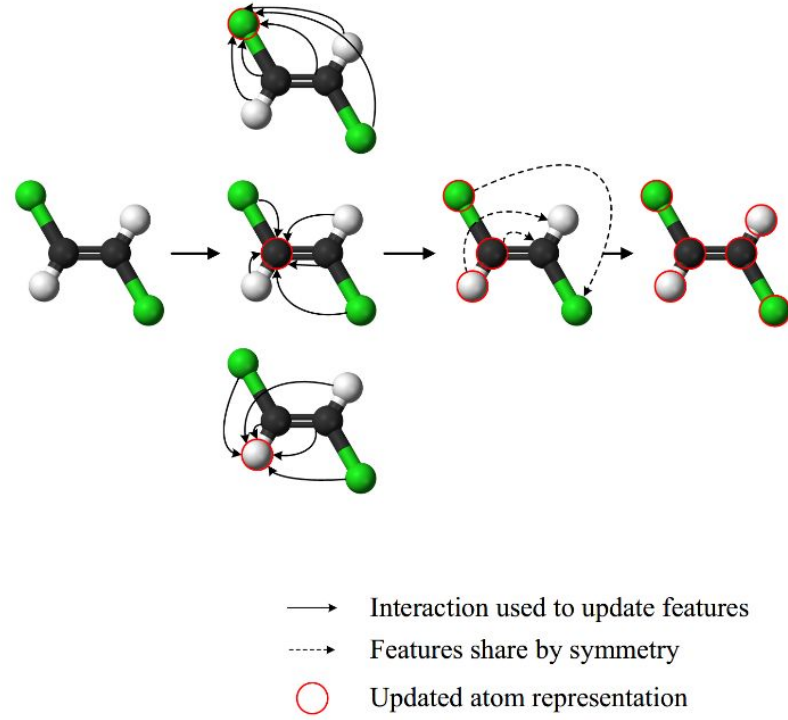

Figure S1. The difference for the model to process with and without symmetry. The figure shows that for trans-1,2-Dichloroethylene with $\mathrm{C}_{2 \mathrm{~h}}$ symmetry, symmetry can help to reduce half redundant interaction calculation.

\begin{tabular}{|c|c|c|c|c|c|c|c|c|c|}
\hline$C_{4 h}$ & $E$ & $C_{4}$ & $C_{2}$ & $C_{4}^{3}$ & $i$ & $S_{4}^{3}$ & $\sigma_{h}$ & $S_{4}$ & linear \\
\hline$A_{g}$ & 1 & 1 & 1 & 1 & 1 & 1 & 1 & 1 & $R_{z}$ \\
\hline$B_{g}$ & 1 & -1 & 1 & -1 & 1 & -1 & 1 & -1 & \\
\hline$E_{g}$ & 1 & $I$ & -1 & $-i$ & 1 & 1 & -1 & $-i$ & $\left(R_{x}, R_{y}\right)$ \\
\hline$A_{u}$ & 1 & $-i$ & -1 & $i$ & 1 & $-i$ & -1 & $I$ & \\
\hline$B_{u}$ & 1 & -1 & 1 & -1 & -1 & 1 & -1 & 1 & \\
\hline$E_{u}$ & 1 & $I$ & -1 & $-i$ & -1 & $-i$ & 1 & $i$ & \\
& 1 & $-i$ & -1 & $i$ & -1 & $i$ & 1 & $-i$ & $(x, y)$ \\
\hline
\end{tabular}

Table S1. Character table of the symmetry group $\mathbf{C}_{4 \mathrm{~h}}$.

\begin{tabular}{|l|l|l|l|} 
Transition & Polarization of light & Tensor product & Decomposition \\
\hline
\end{tabular}




\begin{tabular}{|c|c|c|c|}
\hline \multirow{2}{*}{$T$ номо $\rightarrow L U$ Мо } & $(x, y)$ & $B_{u} \otimes E_{u} \otimes A_{u}$ & $2 E_{u}$ \\
\hline & $z$ & $B_{u} \otimes A_{u} \otimes A_{u}$ & $B_{u}$ \\
\hline \multirow{2}{*}{$T_{\text {HOмо }-1} \rightarrow L U$ LO } & $(x, y)$ & $B_{u} \otimes E_{u} \otimes E_{g}$ & $2 A_{g}+2 B_{g}$ \\
\hline & $z$ & $B_{u} \otimes A_{u} \otimes E_{g}$ & $2 E_{g}$ \\
\hline \multirow{2}{*}{$T_{\mathrm{HOMO}} \rightarrow L U \mathrm{MO}+1$} & $(x, y)$ & $E_{g} \otimes E_{u} \otimes A_{u}$ & $2 A_{g}+2 B_{g}$ \\
\hline & $z$ & $E_{g} \otimes A_{u} \otimes A_{u}$ & $2 E_{g}$ \\
\hline
\end{tabular}

Table S2. Spectral transition probabilities. The tensor product was carried out with the irreducible representations of the initial and final molecular orbital, and the one of the incident light, which has the same linear symmetry as directions $x, y, z$. If the symmetric representation ( $A g$ in this case) appears in the decomposition of the resulting tensor product, the excitation is via incident light with the corresponding linear symmetry.

\begin{tabular}{|c|c|c|c|c|c|c|c|}
\hline \multirow{2}{*}{ Property } & \multirow{2}{*}{ Unit } & \multicolumn{2}{|c|}{$\begin{array}{c}\text { SY-GNN with } \\
\text { sum-up loss }\end{array}$} & \multicolumn{2}{|c|}{$\begin{array}{c}\text { SY-GNN with } \\
\text { Roulette } \\
\text { optimization }\end{array}$} & $\begin{array}{c}\text { MAE } \\
\text { percentage } \\
\text { difference }\end{array}$ & $\begin{array}{c}\text { RMSE } \\
\text { percentage } \\
\text { difference }\end{array}$ \\
\cline { 3 - 6 } & & MAE & RMSE & MAE & RMSE & & \\
\hline alpha & Bohr $^{3}$ & 24.940 & 51.140 & 3.056 & 31.400 & $87.7 \%$ & $38.6 \%$ \\
G & $\mathrm{eV}$ & 1.52375 & 2.10657 & 0.04729 & 0.07089 & $96.9 \%$ & $96.6 \%$ \\
$\mathrm{H}$ & $\mathrm{eV}$ & 1.69855 & 2.42650 & 0.03620 & 0.05762 & $97.9 \%$ & $97.6 \%$ \\
$\mathrm{U}$ & $\mathrm{eV}$ & 1.76778 & 2.19190 & 0.11908 & 0.16926 & $93.3 \%$ & $92.3 \%$ \\
$\mathrm{U} 0$ & $\mathrm{eV}$ & 1.53778 & 2.11324 & 0.03288 & 0.05382 & $97.9 \%$ & $97.5 \%$ \\
$\mathrm{R} 2$ & $\mathrm{Bohr}$ & 10811.0 & 12880.0 & 90.7 & 136.1 & $99.2 \%$ & $98.9 \%$ \\
$\mathrm{mu}$ & $\mathrm{D}$ & 0.00024 & 0.00478 & 0.00002 & 0.00047 & $90.5 \%$ & $90.1 \%$ \\
$\mathrm{CV}$ & $\mathrm{Cal} / \mathrm{mol} \mathrm{K}$ & 3.380 & 4.497 & 0.657 & 0.970 & $80.6 \%$ & $78.4 \%$ \\
ZPVE & $\mathrm{eV}$ & 4.706 & 15.132 & 0.008 & 0.015 & $99.8 \%$ & $99.9 \%$ \\
HOMO & $\mathrm{eV}$ & 0.01518 & 0.02400 & 0.01376 & 0.02089 & $9.4 \%$ & $12.9 \%$ \\
LUMO & $\mathrm{eV}$ & 0.02240 & 0.02681 & 0.01475 & 0.02186 & $34.1 \%$ & $18.5 \%$ \\
gap & $\mathrm{eV}$ & 0.03292 & 0.03891 & 0.01822 & 0.02765 & $44.6 \%$ & $28.9 \%$ \\
\hline
\end{tabular}

Table S3. Comparison for SY-GNN with different optimization methods. The result of SY-GNN with sum-up loss back-propagation and roulette loss back-propagation is showed. It is hard for sumup loss to find suitable parameters to balance so many errors, so some properties will have incredible high loss. And Roulette optimization can avoid this problem and can significantly reduce the loss for all properties. 


\begin{tabular}{|c|c|c|c|c|c|c|}
\hline \multirow{2}{*}{ Type } & \multirow{2}{*}{ Property } & \multirow{2}{*}{ Unit } & \multicolumn{2}{|c|}{ SchNet (MTL) } & \multicolumn{2}{c|}{ SY-GNN } \\
& & & MAE & RMSE & MAE & RMSE \\
\hline ex (R) & $\alpha$ & Bohr $^{3}$ & 1.067 & 1.556 & $\mathbf{0 . 1 2 4}$ & $\mathbf{0 . 2 6 7}$ \\
ex (R) & $\mathrm{G}$ & $\mathrm{eV}$ & 2.47515 & 3.27625 & $\mathbf{0 . 0 6 1 0 4}$ & $\mathbf{0 . 0 8 6 9 8}$ \\
ex (R) & $\mathrm{H}$ & $\mathrm{eV}$ & 2.57257 & 3.53204 & $\mathbf{0 . 0 6 4 7 5}$ & $\mathbf{0 . 0 9 1 1 1}$ \\
ex (R) & $\mathrm{U}$ & $\mathrm{eV}$ & 2.44494 & 3.23544 & $\mathbf{0 . 0 6 3 5 7}$ & $\mathbf{0 . 0 8 9 6 4}$ \\
ex (R) & $\mathrm{U}_{0}$ & $\mathrm{eV}$ & 2.43923 & 3.25176 & $\mathbf{0 . 0 6 4 4 2}$ & $\mathbf{0 . 0 9 0 8 4}$ \\
ex (R) & $\mathrm{R}^{2}$ & $\mathrm{Bohr}^{2}$ & 32.5 & 45.7 & $\mathbf{2 . 5}$ & $\mathbf{9 . 9}$ \\
ex (R) & $\mu$ & $\mathrm{D}$ & $\mathbf{0 . 0 2 2 5 3}$ & $\mathbf{0 . 0 6 1 1 9}$ & 0.14270 & 0.20320 \\
ex (R) & $\mathrm{C}_{\mathrm{V}}$ & $\mathrm{Cal} / \mathrm{mol} \mathrm{K}$ & 0.465 & 0.595 & $\mathbf{0 . 0 6 2}$ & $\mathbf{0 . 0 9 5}$ \\
in (K) & ZPVE & $\mathrm{eV}$ & 0.1797 & 0.2860 & $\mathbf{0 . 0 0 3 6}$ & $\mathbf{0 . 0 0 4 9}$ \\
in (K) & $\epsilon_{\text {Hомо }}$ & $\mathrm{eV}$ & 0.3088 & 0.4057 & $\mathbf{0 . 0 5 5 3}$ & $\mathbf{0 . 0 7 5 6}$ \\
in (K) & $\epsilon_{\text {LUMO }}$ & $\mathrm{eV}$ & 0.6561 & 0.7989 & $\mathbf{0 . 0 5 2 3}$ & $\mathbf{0 . 0 7 3 1}$ \\
in (K) & gap & $\mathrm{eV}$ & 0.7684 & 0.9374 & $\mathbf{0 . 0 7 1 6}$ & $\mathbf{0 . 1 0 0 0}$ \\
\hline
\end{tabular}

Table S4. QM9 results. The result of SchNet (MTL) and SY-GNN on QM9 is showed. QM9 is a widely used benchmark dataset, which contains molecules with most 9 heavy atoms.

\begin{tabular}{|c|l|l|}
\hline Property & MAE & RMSE \\
\hline$\epsilon_{\text {HOMO }-5}$ & 0.1527 & 0.4066 \\
$\epsilon_{\mathrm{HOMO}}-4$ & 0.1530 & 0.4128 \\
$\epsilon_{\mathrm{HOMO}}-3$ & 0.1275 & 0.3660 \\
$\epsilon_{\mathrm{HOMO}}-2$ & 0.0805 & 0.2991 \\
$\epsilon_{\mathrm{HOMO}}-1$ & 0.0745 & 0.2946 \\
$\epsilon_{\mathrm{LUMO}}+1$ & 0.0681 & 0.3199 \\
$\epsilon_{\mathrm{LUMO}}+2$ & 0.0985 & 0.3342 \\
$\epsilon_{\mathrm{LUMO}}+3$ & 0.0844 & 0.3068 \\
$\epsilon_{\mathrm{LUMO}}+4$ & 0.1106 & 0.3548 \\
$\epsilon_{\mathrm{LUMO}}+5$ & 0.1431 & 0.4104 \\
\hline
\end{tabular}

Table S5. Orbital energy prediction results. The results of the energy of orbits prediction using SY-GNN method are shown (Unit: eV). As we all know, there are no other DNN methods that shows the results of orbit energy prediction besides HOMO, LUMO. The results are training individually. All properties in the table are intensive and in $\mathrm{K}$ space. 


\section{References}

1. Schutt, K.; Kessel, P.; Gastegger, M.; Nicoli, K.; Tkatchenko, A.; Muller, K.-R., Schnetpack: A Deep Learning Toolbox for Atomistic Systems. Journal of chemical theory and computation 2018, 15, 448-455.

2. Paszke, A.; Gross, S.; Massa, F.; Lerer, A.; Bradbury, J.; Chanan, G.; Killeen, T.; Lin, Z.; Gimelshein, N.; Antiga, L. In Pytorch: An Imperative Style, High-Performance Deep Learning Library, Advances in Neural Information Processing Systems, 2019; pp 8024-8035.

3. Kingma, D. P.; Ba, J., Adam: A Method for Stochastic Optimization. arXiv preprint arXiv:1412.6980 2014.

4. Lu, C.; Liu, Q.; Wang, C.; Huang, Z.; Lin, P.; He, L., Molecular Property Prediction: A Multilevel Quantum Interactions Modeling Perspective. In The Thirty-Third AAAI Conference on Artificial Intelligence, 2019; pp 1052-1060.

5. Tsubaki, M.; Mizoguchi, T., Correction to "Fast and Accurate Molecular Property Prediction: Learning Atomic Interactions and Potentials with Neural Networks". The journal of physical chemistry letters 2019, 10, $2066-2067$. 